\title{
Téoros
}

Revue de recherche en tourisme

\section{Tourisme, marginalité sociale et restauration du centre historique de la ville de Salvador au Brésil}

\section{Marc Perreault}

Volume 24, numéro 3, automne 2005

Tourisme et santé, quelques perspectives

URI : https://id.erudit.org/iderudit/1071079ar

DOI : https://doi.org/10.7202/1071079ar

Aller au sommaire du numéro

Éditeur(s)

Université du Québec à Montréal

ISSN

0712-8657 (imprimé)

1923-2705 (numérique)

Découvrir la revue

Citer cet article

Perreault, M. (2005). Tourisme, marginalité sociale et restauration du centre historique de la ville de Salvador au Brésil. Téoros, 24(3), 37-44.

https://doi.org/10.7202/1071079ar d'utilisation que vous pouvez consulter en ligne. 


\section{Tourisme, marginalité sociale et restauration du centre historique de la ville de Salvador au Brésil}

\begin{abstract}
Marc Perreault
Le tourisme est souvent perçu comme la panacée aux problèmes de développement économique qui affectent certaines régions. L'industrie touristique est, en effet, dans un monde qui valorise les loisirs et la consommation, un des secteurs de l'économie qui connaît depuis quelques décennies une des plus fortes croissances ${ }^{1}$. Dans certains pays, dont un grand nombre dit «en développement ", elle est non seulement la première industrie, mais une des principales façons d'attirer les capitaux étrangers. Aussi, à première vue, est-il justifié de développer ce secteur particulier de l'économie, en pensant que ses retombées pourront profiter tout autant aux populations locales qu'à la prospérité des régions et du pays. Mais en est-il toujours vraiment ainsi ? Une vision strictement économique du tourisme est-elle par définition compatible avec les enjeux sociaux que, parfois, au niveau des populations locales, on souhaite ou prétend pouvoir régler avec les capitaux et les infrastructures de cette industrie?
\end{abstract}

À partir de l'exemple de la restauration à des fins touristiques du centre historique de la ville de Salvador au Brésil, nous dégagerons dans cet article quelques-unes des limites au développement touristique vu en termes de solution aux problèmes sociaux qui affectent les populations concernées par ce développement. Si le tourisme a indéniablement des retombées positives sur l'économie, il est aussi générateur de son lot de problèmes qui peuvent se répercuter sur la santé globale des populations. La marginalité sociale est l'un de ces problèmes. Entre autres, dans les pays en développement, le tourisme crée dans son sillon de nouvelles formes de dépendance auprès de la population locale. Comme l'ont souligné Boutillier, Fieloux et Ormières (1978)

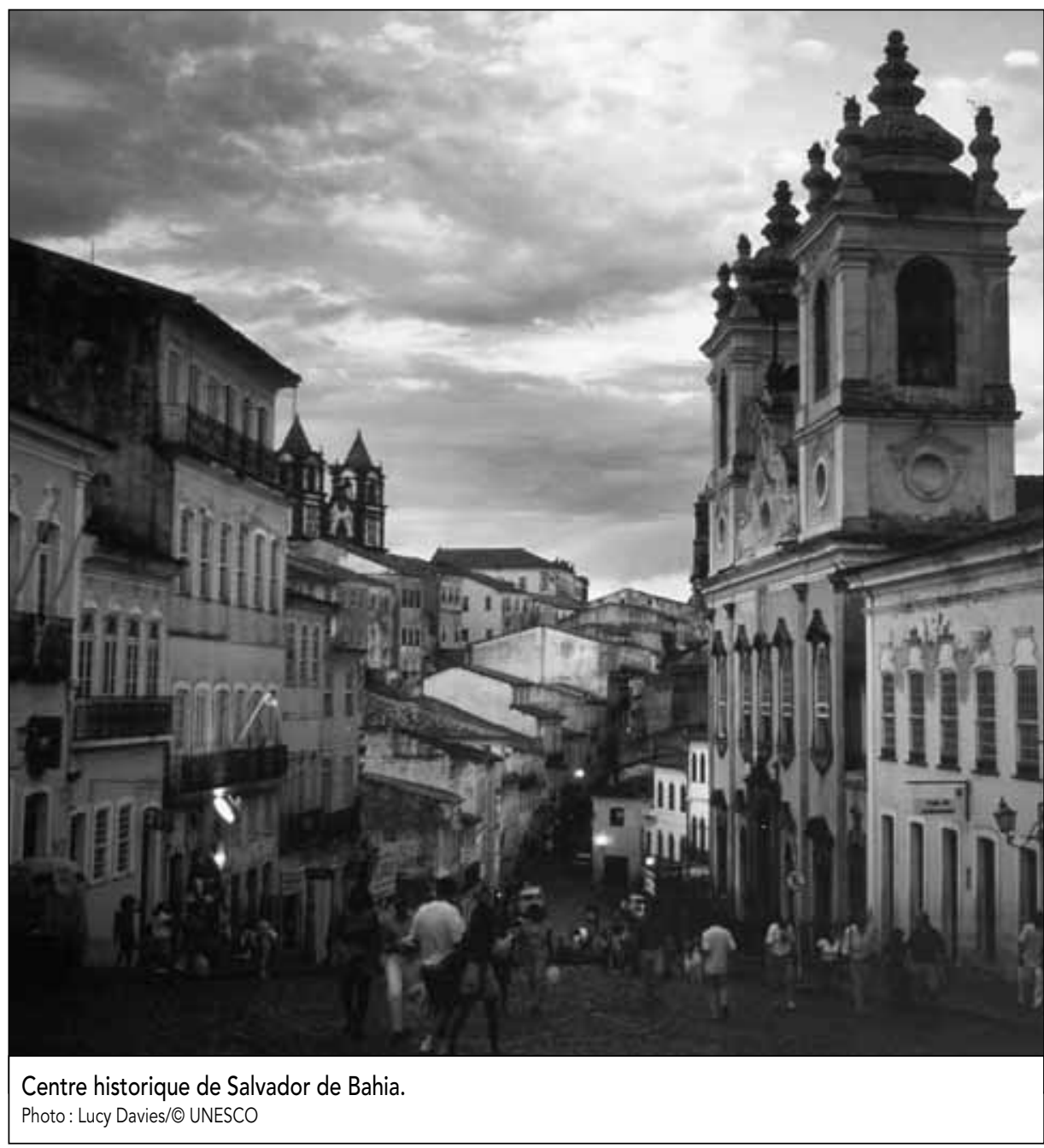

pour l'Afrique de l'Ouest, les zones où se concentrent les touristes étrangers favorisent l'émergence de "parasites sociaux " et de la mendicité. Non seulement l'émergence de catégories de "parasites sociaux " peut-elle conduire à une certaine instabilité sociale et devenir à la longue un problème politique, mais, aussi, ajoutent les auteurs (1978: 65), "la présence de touristes à un niveau de consommation très élevé, en soulignant les différences de revenus entre les groupes sociaux, peut éveiller certaines tensions ".

Avec ses conséquences positives ou néfastes sur les populations locales, le tourisme s'avère dans certaines régions un vecteur important de «santé ». La notion de santé ne doit pas être comprise ici strictement comme un état de bien-être personnel, même si, comme on le verra, elle peut aussi jouer en cette ma- 
tière un rôle important, mais elle doit être comprise surtout dans ses dimensions globales et communautaires. En fait, pour une majorité de population, en particulier dans les sociétés dites traditionnelles, la notion moderne et occidentale de santé individuelle n'existe pas ou, du moins, n'est pas dominante. Ce que nous appelons "santé " correspond plus ou moins dans ce type de société à un état de bien-être ou d'équilibre communautaire qui concerne non seulement la personne au sein de sa famille, mais aussi la personne dans ses relations globales avec sa communauté, son milieu et, par extension, avec l'ensemble du cosmos. Aussi, lorsqu'une personne est " malade», se doit-on, dans ces sociétés, de la traiter en prenant en considération l'ensemble des relations d'interdépendance qui la relient à son environnement. Une telle vision " holistique » de la santé suppose de même, à l'inverse, que les éléments sociaux, environnementaux et cosmiques peuvent affecter la santé individuelle des personnes et être des « vecteurs » de maladie.

Dans le présent article, nous nous intéressons plus particulièrement aux effets que le développement touristique, en l'occurrence ici la restauration du centre historique de la ville de Salvador, peut avoir sur les dynamiques de la marginalité sociale et de la violence urbaine ${ }^{2}$. L'exemple de Salvador est éclairant en ce sens qu'un des motifs avoués de la restauration à des fins touristiques était de pouvoir contrer, sinon éliminer carrément, la violence et la marginalité urbaines qui sévissaient dans le secteur avant la revitalisation. Or, plus de treize ans après le début des travaux, force est de constater que non seulement les problèmes de marginalité sociale sont toujours présents, mais ceuxci se sont aujourd'hui complexifiés avec les nouvelles réalités sociales engendrées, entre autres, par la restauration du centre historique, patrimoine mondial de l'Organisation des Nations unies pour l'éducation, la science et la culture (Unesco).

Cet article est avant tout un texte de réflexion. II se fonde pour l'essentiel sur des données empiriques de recherche que nous avons recueillies de 1983 à 2004 lors de différents travaux portant sur les milieux marginaux du centre historique de Salvador. S'ajoute à cette perspective temporelle sur la transformation des groupes marginaux de jeunes, la révision du processus historique et des enjeux sociaux qui sont à l'origine de la restauration du centre historique à des fins touristiques. La question de recherche à la base de notre réflexion est : « Jusqu'à quel point le développement touristique peut-il ou non se faire sans la participation ou, à tout le moins, l'inclusion de l'ensemble des groupes qui composent la population locale affectée par ce développement? "Nous ne prétendons pas dans cet article pouvoir répondre de manière définitive ni exhaustive à cette importante question. Cependant, nous souhaitons mettre en relief les limites d'une vision strictement économique du développement touristique vu, dans ce cas-ci, comme une solution à certains problèmes sociaux. De manière générale, nous cherchons également par cet article à soulever les limites de la restauration du cadre bâti comme réponse possible à l'élimination de la marginalité sociale, sinon à son expulsion vers d'autres lieux ${ }^{3}$.

Après avoir dégagé quelques-unes des particularités touristiques de Salvador, nous présentons les principales étapes du long processus qui a conduit à la revitalisation de son centre historique à des fins essentiellement touristiques. Nous terminons avec quelques observations sur les impacts de cette restauration sur la marginalité sociale et la population environnante.

\section{Salvador de Bahia, une industrie touristique fondée sur la fête}

Voyage et Brésil riment à l'oreille de plusieurs avec Rio, son Pain de Sucre, ses plages bondées de corps huilés qui se font brunir au soleil, ses écoles de samba et son carnaval aussi exubérant que coloré. Évidemment, le cinquième plus grand pays en superficie de la planète, peuplé de ses 180 millions d'habitants, ne saurait se résumer à la seule réalité touristique de la «belle » Rio de Janeiro. L'Amazonie est une autre région du Brésil qui éveille chez le touriste voyageur, en quête d'exotisme et de paradis perdu, un tout autre type de fantasmagorie. Mais, hormis les paysages fabuleux, tels que les chutes d'Iguaçu qui captivent l'imaginaire des touristes vacanciers, c'est surtout le «style brésilien ", avec ses musiques, son futebol, la démesure de ses célébrations festives, qui pousse le touriste à l'esprit quelque peu aventurier à choisir cette destination plutôt qu'une autre. Or, peu de gens à l'extérieur du Brésil savent que le carnaval de Rio, symbole par excellence de ce «style brésilien », a été supplanté depuis quelques années, tant en participation qu'en intensité, par celui de la ville de Salvador. La première capitale de I'histoire du pays attire, en effet, chaque année, un nombre toujours croissant de touristes, pour une large part brésiliens, mais provenant de plus en plus des quatre coins du monde. Dans ce pays à forte compétition régionale, vestige probablement des rivalités entre les anciennes oligarchies, la ville de Salvador se targue d'avoir le carnaval « No 1 » non seulement du Brésil, mais de la planète entière. Aussi, aime-t-on répéter durant les festivités, que le carnaval de Salvador est la plus grande fête populaire de rue au monde!

La ville de Salvador, de son nom complet Salvador de la baie de tous les saints (Bahia de Todos os Santos), ou simplement Bahia ${ }^{4}$, comme l'appellent affectueusement ses habitants, a su se faire une réputation qui dépasse les frontières du Brésil, grâce à son héritage africain et à l'atmosphère de fête permanente qui y règne. Cette réputation, aussi surfaite qu'elle soit, n'a pas que de bons côtés. Elle devient même dans certaines situations prétexte à la stigmatisation du Bahianais que l'on aime décrire ailleurs au Brésil, en particulier dans les grands centres du sud du pays, comme le Noir pétulant, figé dans la «tradition », qui ne pense qu'à fêter et à s'amuser plutôt qu'à travailler, le travail étant une valeur associée au "progrès" dans les représentations sociales de ce pays qui a adopté pour devise la formule "ordre et progrès » du philosophe Auguste Comte. Mais, quoi qu'il en soit, la fête est un produit qui se vend bien et elle est devenue une, sinon la principale carte d'attraction de l'industrie touristique locale. On peut lire, sur les multiples sites promotionnels Internet, autant de la ville de Salvador que de l'État de Bahia: "La Bahia est toujours en fête ${ }^{5}$ ! »

II faut dire que la réputation festive de « la Bahia " ne date pas d'hier. Les voyageurs étrangers sont d'ailleurs du nombre de ceux qui ont contribué à forger cette réputation. Par exemple, cette description, un brin exagérée, laissée par le voyageur britannique E.F. Knight après son passage à Salvador dans la seconde moitié du XIX ${ }^{e}$ du siècle:

À Bahia, chaque jour de l'année semble être une fête dédiée à l'un ou l'autre saint. Fêter un jour saint implique ici une grande dépense en feux d'artifice en plus de faire sonner les cloches de l'église. Les feux éclatent toute la journée puisque personne n'attend jusqu'à la nuit pour les lancer. II n'y a aucune place 
au monde comparable à Bahia pour ce type de divertissement. Au loin, de la mer, on peut savoir que l'on approche de ce port à cause du bruit et de l'éclat des marques d'adoration de ces habitants. (Cité dans Verger, 1981 : 99, traduction libre.)

Cette propension à la fête se traduit dans l'œuvre du sociologue et écrivain Gilberto Freyre comme un trait identitaire du peuple bahianais. À son tour, il écrit, en 1934: "À Bahia, on a l'impression que tous les jours sont des jours de fêtes. Fêtes d'églises brésiliennes, avec leurs ornements de roseaux, leurs gâteaux, leurs feux d'artifice, leurs amourettes » $(1974:$ 268). Or, cette inclination pour la fête serait d'abord et avant tout le propre de l'héritage africain de la population. Ainsi, Freyre oppose l'introversion de l'Indien à l'extroversion de l'Africain: "Le comportement des populations négroïdes comme celle de Bahia, gaie, expansive, sociable, bavarde " se distingue, explique-t-il, de celui des « autres moins influencées par l'apport de sang nègre et davantage par celui de l'indigène » (idem : 267).

Populations tristes, silencieuses, repliées sur elles-mêmes, taciturnes, voilà ce que sont les populations de l'extrême Nord et de l'intérieur, sans l'allégresse communicative des gens de Bahia, sans leur pétulance parfois irritante. Mais aussi sans leur charme, leur spontanéité, leur courtoisie, leur bon rire contagieux (idem: 267-268).

Au-delà de son côté caricatural, la description de Freyre a joué un rôle important en matière d'intégration de la composante africaine dans la conception moderne de l'identité brésilienne. Au moment où Freyre écrivait ces lignes, les élites brésiliennes cherchaient toujours un moyen de surmonter le « retard brésilien », fruit de quatre siècles d'esclavage et de la présence de ces citoyens de seconde zone que constitue la population de descendance africaine. Le blanchiment de la population semblait, pour une majorité, être alors la seule voie possible vers le «progrès ". La thèse de Freyre, selon laquelle le Brésilien est né du mélange racial entre le Portugais, I'Indien et l'Africain, allait permettre à ce dernier, qui était auparavant exclus de représentations positives de l'identité brésilienne, de ne plus être vu seulement en termes négatifs. Cette valence identitaire positive, le descendant africain l'incarnera plus spécia- lement par son apport à l'esprit festif de la société brésilienne. Or, faut-il le souligner, lorsqu'il n'est pas associé à cette esthétique festive ou valorisé par ses qualités sportives, le « Noir » est plus souvent qu'autrement encore représenté en termes de « retard social »; la pauvreté, la violence urbaine, la criminalité, entre autres, étant des «phénomènes » très associés, dans l'imagerie populaire, à la couche noire de la population.

L'univers religieux afro-brésilien n'est pas non plus étranger à la réputation festive de Bahia. Souvent décrite comme une ville africaine en Amérique, Salvador est le principal berceau du candomblé, religion populaire d'origine africaine dominée par les rythmes des tambours et les chorégraphies colorées des fidèles. La prégnance mystique du candomblé est telle que dès la fin du XIXe siècle, chercheurs brésiliens d'abord, anthropologues de partout ailleurs dans le monde ensuite, sont venus y chercher l'Afrique mythique des origines. Ainsi, avait-on l'impression de retrouver en terre brésilienne des pratiques religieuses africaines disparues sur le continent. À cette époque débutent également des voyages de ressourcement entre la côte de Bahia et la côte du Bénin. Prêtres ou prêtresses du candomblé ${ }^{6}$ se rendent en Afrique pour se réapproprier un savoir et des pratiques qui leur permettront de solidifier les bases de leur culte alors en émergence. À partir du milieu des années 1950, ces voyages entre Bahia et l'Afrique s'intensifieront grâce à l'alliance inusitée des anthropologues avec les leaders de culte. Non seulement les Brésiliens se rendent-ils en Afrique pour se ressourcer, mais les Africains viennent à Salvador pour se réapproprier des éléments de culte oubliés. Le prestige des leaders du candomblé de Bahia fera que l'on viendra ensuite de partout au Brésil, mais aussi d'ailleurs en Amérique latine, apprendre de leur savoir, reconnu comme le «plus pur et le plus traditionnel ${ }^{7}$ ».

Les « lavages » publics des églises sont certainement, en dehors du carnaval proprement dit, parmi les types de fêtes populaires qui contribuent le plus à faire mousser la réputation festive de Bahia parmi tout le Brésil. Fête qui n'était pas sans rappeler, à l'origine, la «messe des fous » de l'Europe médiévale ; les «lavages » concrétisent la rencontre burlesque entre les univers religieux catholiques et afro-brésiliens et celui de la fête carnavalesque. Alors qu'auparavant on «la- vait » l'église en entier, aujourd'hui on ne "lave" plus que le parvis, les autorités ecclésiales voyant d'un mauvais œil l'esprit de licence libertine qui s'emparait des festivaliers alors qu'ils envahissaient l'enceinte sacrée de leur lieu de culte. Véritables fêtes de quartiers, la popularité des «lavages» est telle, aujourd'hui, que l'on invente à chaque année de nouveaux lavages à des fins strictement commerciales. Dans ces cas, on pourra laver tout, autant les berges de la mer qu'une place publique. Ainsi, le sens originel des «lavages » se perd avec sa commercialisation. Les fêtes du lavage - dont la plus grande est celle de l'Église du Bonfim qui a lieu le deuxième jeudi après l'épiphanie-, lesquelles rassemblent des centaines de milliers de fêtards, s'inscrivent dans le cycle carnavalesque des fêtes de renouvellement de l'année, de telle sorte qu'à Salvador les festivités carnavalesques débutent littéralement le 2 décembre pour se terminer dans l'apothéose que constitue la semaine du carnaval, que l'on cherchera à prolonger bien au-delà du Mercredi des Cendres.

Mais si Salvador a aujourd'hui gagné le " concours " (non officiel, mais très présent) du plus grand carnaval du Brésil, voire du monde entier, c'est grâce à la popularité contagieuse des "trios électriques", ces énormes camions sur lesquels sont juchés des orchestres qui font vibrer aux rythmes de leur musique les milliers de festivaliers qui les suivent dans un pas de danse endiablé aux limites de la transe (collective) et du pugilat. Durant le carnaval, ils seront plus de deux millions de personnes dans les rues à suivre nuit et jour, presque sans interruption, leur " trio » favori. Le spectacle de cette foule en délire est si impressionnant qu'il est aujourd'hui retransmis en direct à la télévision dans tout le Brésil. Aussi le phénomène des trios électriques s'est-il répandu avec le temps à d'autres régions du pays, voire même à d'autres régions du monde.

En dehors de ces fêtes de la démesure qui se déroulent à différents moments de l'année aux quatre coins de la ville, les promoteurs urbains et touristiques ont choisi de faire du centre historique, en particulier du Pelourinho $^{8}$, le haut lieu de la fête permanente à Salvador. Pour ce faire, il a fallu non seulement entreprendre de grandioses travaux de restauration, mais il aussi fallu « réafricaniser » littéralement cet espace urbain qui, jusqu'au milieu des années 1980, vibrait 
aux rythmes de la pop américaine plutôt qu'à ceux des musiques reggae ou afro-brésilienne comme c'est le cas aujourd'hui. Le chanteur et auteur-compositeur américain Paul Simon a contribué pour une large part à donner une envergure internationale à ce processus de « réafricanisation » en venant y tourner, à la fin des années 1980, un vidéoclip avec la bande internationale Olodum, un groupe afro-brésilien de percussions qui a depuis installé ses pénates au Pelourinho. Plus tard ce sera à la star américaine Michael Jackson de venir y tourner un vidéoclip, toujours au même endroit et avec le même groupe!

Le centre historique de Salvador appartient au patrimoine mondial de l'Unesco depuis 1985. Le site est porteur d'une histoire riche qui s'échelonne sur plus de cinq siècles, soit depuis la «découverte» du Brésil par les Portugais. Le site est aussi reconnu depuis les premières décennies des années 1900 comme la «zone» (principale) de prostitution de la ville de Salvador avec tout son lot de voyous - malandros - et autres marginaux propres à ces milieux. Le grand écrivain brésilien Jorge Amado raconte dans plusieurs de ses romans, dont Suor ${ }^{9}$ publié en 1934, la vie truculente du petit peuple qui vivait au Pelourinho à cette époque. Or, déjà dans ces années, le tableau que brosse Jorge Amado ne laisse pas de doute quant à l'ambiance malfamée et à l'état d'abandon de l'endroit ${ }^{10}$. À la suite de la visite d'une première délégation de l'Unesco en 1966, on lance concrètement le projet de revitalisation du centre historique ${ }^{11}$ (Miranda et Santos, 2000 : 18) et l'année suivante, sous l'égide de la BID (Banque interaméricaine de développement) et de l'Unesco, on amorce une étude sur la faisabilité économique d'un tel projet. Dans leur rapport, les auteurs de la recherche relèvent notamment l'importance de restaurer le cadre bâti du Pelourinho en guise de réponse aux « problèmes » de prostitution et de drogue (essentiellement du cannabis) dont on dit qu'ils sont en expansion (Braga, 1969). Ainsi, en inscrivant le centre historique de Salvador sur la liste du Patrimoine de l'humanité, I'Unesco souhaite avant tout s'assurer "d'enrayer le processus urbanistique dangereux qui menaçait le centre ", en reconnaissant tout autant la valeur architecturale du site que sa valeur culturelle qui s'exprime dans la « convergence des cultures européennes, africaines et amérindiennes du XVIe au XVIII siècle » ${ }^{12}$.
La première pelletée de terre des travaux tant attendus de restauration n'aura toutefois lieu qu'en 1992. Non seulement le "processus urbanistique dangereux " se manifestait-il alors dans la détérioration extrême du cadre bâti, mais aussi dans la violence urbaine qui sévissait dans tous les environs du Pelourinho et qui faisait fuir, un à un, les touristes qui étaient les premiers à en faire les frais. Nous avons personnellement écopé de cette violence. De retour en 1991, après près de cinq ans d'absence, nous avons été attaqué dans l'entrée de notre hôtel le soir même de notre arrivée par une douzaine de jeunes. Question de nous rassurer sur la banalité de cette attaque, des touristes qui ont assisté impuissants à la scène, perchés à la fenêtre de leur chambre, nous ont raconté le lendemain avoir vécu pareille mésaventure deux ou trois fois pendant la première semaine de leur séjour! Après les touristes, les premiers à se plaindre ouvertement de cette violence omniprésente étaient les marchands locaux qui voyaient, impuissants, leurs affaires péricliter $^{13}$. La situation était tout autre lorsque y sommes retourné au début de l'année 1996. Dans ce secteur, dorénavant sous haute surveillance policière, nous avions l'impression, à chaque jour, de découvrir de nouvelles rues, tellement la transformation des lieux était radicale. Les touristes comme les membres de la classe moyenne locale, qui avaient également déserté l'endroit depuis très longtemps, étaient revenus en grande pompe pour fêter et consommer au grand plaisir des commerçants, tout sourire, qui bénéficiaient de cette manne inespérée. L'opération revitalisation semblait être un véritable succès. Les marginaux sociaux, bloqués dès leur entrée par les nombreuses guérites de contrôle que l'on avait installées, ne pénétraient plus les rues du Pelourinho. L'ambiance était à la fête et l'argent affluait dans les coffres des investisseurs. L'avenir était prometteur.

\section{La restauration : une saga longtemps annoncée}

Avant toute chose, afin qu'il n'y ait pas d'ambiguïtés sur le sens de notre propos qui concerne strictement les aspects humains et sociaux de la revitalisation, spécifions que le résultat des travaux (toujours en cours) de restauration des places et des immeubles du centre historique est tout à fait remarquable. II était devenu dangereux de vivre dans le secteur non pas juste à cause de la violence, mais parce que la majorité des immeu- bles tombaient littéralement en ruine. II nous fallait même parfois enjamber deux marches absentes pour nous rendre à l'étage supérieur où s'entassaient, dans des chambres minuscules, familles, enfants, prostituées ou autres marginaux. Des murs entiers de maisons à étage s'étaient écroulés ou en étaient sur le point. Les autres façades d'immeubles encore debout étaient, quant à elles, complètement défraîchies et verdies par la moisissure accumulée par les années d'air marin. L'hygiène, tant des commerces que des lieux de vie de plusieurs ménages, était souvent déficiente du fait de l'absence de salle d'eau et même de toilettes. Cela dit, nous gardons le souvenir que les personnes qui vivaient dans ces endroits étaient très fières; la plupart d'entre elles ne sortaient dans la rue que vêtues de vêtements impeccablement propres et toujours bien pressés! Avant toute chose, le Pelourinho c'était, à l'époque, une ambiance de vie fondée sur l'allégresse contagieuse de ses habitants et non pas un endroit où l'on se rendait d'abord pour la beauté des lieux.

Or, s'il n'y a aucun doute que la restauration était devenue absolument nécessaire, on est en droit cependant de s'interroger sur la manière dont elle s'est faite. La première critique que l'on peut adresser concerne le temps qui s'est écoulé entre le moment où le projet de revitalisation a été annoncé comme étant incontournable et le moment où les travaux ont débuté. On parle ici de plus de vingt ans pendant lesquels le cadre bâti des immeubles s'est détérioré à une vitesse d'autant plus accélérée que les propriétaires et les promoteurs, connaissant les intentions d'expropriation du secteur par les autorités en place, ont laissé leurs immeubles à l'abandon sachant qu'ils seraient de toute façon largement compensés en temps et lieu. Conséquence en grande partie de cette détérioration extrême des lieux, l'autre critique que nous pouvons adresser et qui nous apparait la plus déterminante, est le fait que la restauration n'ait pu se faire sans l'expropriation de l'ensemble des habitants du secteur. II était entendu que la population pauvre et marginale n'avait pas sa place dans le nouveau Pelourinho. En fait, le succès économique et touristique de cette entreprise grandiose dépendait beaucoup, aux yeux de ses principaux promoteurs, du nettoyage du secteur de tous ces indésirables qui y vivaient ou y vivotaient et que plusieurs tenaient, faute d'autres émissaires visibles, pour responsables du laisser à l'abandon des lieux et, 
surtout, de la violence. La nouvelle vocation du Pelourinho et de ses environs n'était plus résidentielle, mais strictement économique. D'aucuns diront que l'on a voulu créer une sorte de «Dysneylandia » de la culture afro-brésilienne où tout prend la forme d'un produit de consommation qui se monnaye à fort prix. Dans cet univers de bistrots-terrasses avec scènes de spectacles, de restaurants onéreux et de boutiques de souvenirs de toutes sortes, seule importait au début la venue de nouveaux investisseurs avec leurs capitaux. Les anciens résidants des lieux n'y avaient d'autant plus leur place que le coût de la vie aux abords du Pelourinho était devenu, en cette période de redressement de la monnaie brésilienne, tout à fait inabordable non seulement pour les pauvres, mais pour tout ménage brésilien à revenu moyen. En fait, à l'exception des derniers édifices en attente d'être évacués, et qui sont pour la plupart tous occupés par des squatters plus ou moins légalement tolérés, il n'y a plus aucune famille qui vit dans les immeubles rénovés du centre historique. Aujourd'hui, alors que la situation a passablement changé et que les groupes et les marginaux sont de retour dans et autour du Pelourinho, les promoteurs commencent à s'interroger sur la viabilité d'un quartier sans habitants. On envisage l'alternative d'encourager les artistes et les jeunes professionnels à venir s'y installer, mais on peut parier que cette solution risque peu de fonctionner, avec le retour en force de la marginalité sociale et de la criminalité liées, surtout, au commerce du crack qui fait des ravages dans le secteur.

Le pari de tenir les marginaux à distance du Pelourinho était certes quelque peu utopique. Pour ce faire, on a d'abord augmenté de façon considérable la présence policière, une police plus active comparé à auparavant, alors que celle-ci préférait fermer les yeux sur la délinquance et la petite criminalité plutôt que d'intervenir. On a même créé un nouveau type de corps policiers dédié spécifiquement au tourisme. À cela s'ajoutait l'embauche de plusieurs gardes de sécurité qui vérifiaient les entrées et les sorties des principaux accès au Pelourinho. Plusieurs anciens résidants du secteur se voyaient ainsi refusé l'accès au milieu de vie qui avait été le leur pendant des décennies, sous prétexte qu'il représentait un risque pour la sécurité publique ${ }^{14}$. Refoulées juste aux limites du Pelourinho, ces personnes exprimaient parfois haut et fort leur colère et leurs frustrations par des menaces. Dans tous les cas, elles restaient actives en périphérie du Pelourinho et n'abandonnaient pas leurs activités illégales, le cas échéant. Ainsi, en dehors du périmètre hautement protégé, il était toujours aussi, sinon plus, dangereux de se promener dans les environs. Avec le temps, et avec la création entre autres de la Place du Reggae ${ }^{15}$, les contrôles de surveillance se sont relâchés et les personnes autrefois exclues ont pu regagner les rues où se déroule l'action principale. Plus cachées ou sournoises, la criminalité comme la petite délinquance sont peu à peu réapparues au Pelourinho.

\section{Commercialisation et effets pervers de la restauration}

Le plus frappant pour quiconque a connu le Pelourinho avant et après la restauration est l'omniprésence aujourd'hui des commerces de facture touristique en contraste avec la spontanéité de la vie populaire de rue qui y régnait auparavant. Dans ce ghetto touristique qu'est devenu le Pelourinho, les autorités locales se doivent de tout contrôler. La rue et les places publiques ne sont le théâtre de la fête que dans un cadre strictement délimité par les autorités municipales et touristiques, alors que la caractéristique principale des fêtes de rue brésiliennes a toujours été leur côté improvisé voire, même, spontané. L'exemple le plus éloquent de cette nouvelle réalité est la transformation de la Fête de la bénédiction du mardi soir qui attire aujourd'hui des dizaines de milliers de personnes provenant d'un peu partout dans Salvador. Cette fête, dont l'organisation était autrefois tout à fait informelle et improvisée, est maintenant entièrement régie par les autorités municipales. Rien n'est laissé sans contrôle, de la phase d'érection des scènes de spectacles jusqu'à l'heure où ceux-ci doivent cesser, en passant par l'esthétique aseptisée des « baraques » où l'on vend bières, alcools et autres rafraîchissements, de même que qui peut ou non vendre ses différents produits de consommation. Au nom de l'idéologie hygiéniste qui revient en force dans les modèles de contrôle de santé publique du Brésil, c'est tout le pan informel et improvisé de la fête qui s'est aujourd'hui écroulé. Ainsi à Salvador, lors de toutes les principales fêtes publiques, s'affairent en nombre et en force les fonctionnaires municipaux de la «fiscalisation " qui s'assurent que tous les commerçants sont en règle, c'est-à-dire dotés des permis et des autorisations leur permettant de vendre leurs produits. Or, la fête, pour le petit peuple, a toujours été une occasion de gagner quelques sous en participant à ce que l'on appelle au Brésil l'« économie pa-

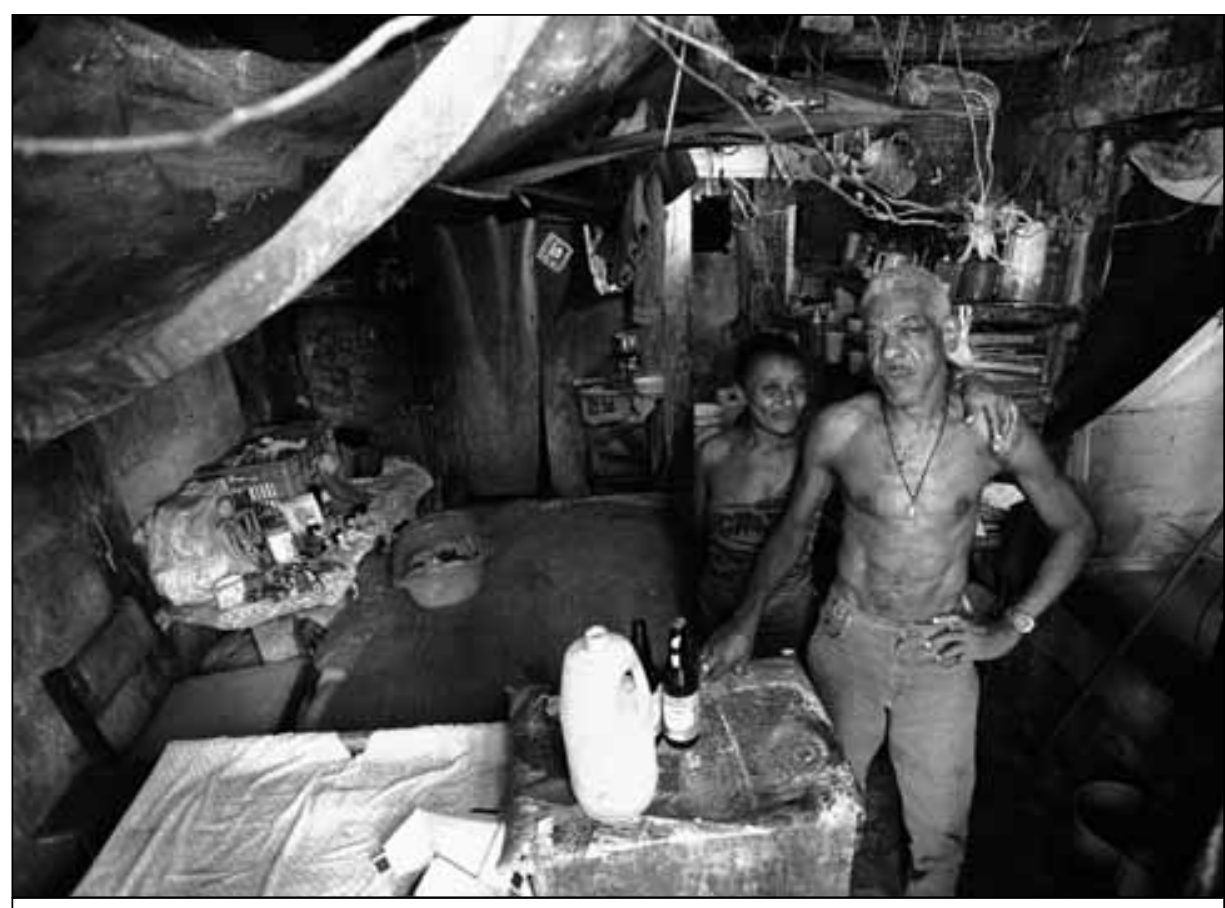

Couple dans leur maison, Salvador de Bahia, Brésil.

Photo: Mélissa Robertson 
rallèle ». Aujourd'hui, le coût et la difficulté d'obtenir les permis nécessaires à la vente dans la rue et/ou lors des événements publics ont mis un frein important à cette économie de la survie. Combien de fois avonsnous vu des petits commerçants ambulants, presque aux larmes, supplier la « police fiscale " de leur laisser la marchandise durement acquise qu'elle venait de leur saisir parce que tout n'était pas en règle?

Mais jusqu'à quel point l'abondance de contrôles sociaux, fiscaux, hygiéniques ou policiers - peu importe en fait leur forme peut-elle vraiment assurer la sécurité du public ? L'exemple du ghetto touristique qu'est devenu le Pelourinho au centre historique de Salvador devrait pouvoir nous faire réfléchir sur les limites et les effets pervers de tels contrôles.

Si le périmètre du Pelourinho demeure encore relativement sécuritaire, conséquence de la présence policière qui fait de l'endroit presque un état policier, il est cependant de nos jours beaucoup plus dangereux qu'auparavant de circuler hors des limites de cette zone protégée. En fait, même à l'intérieur du Pelourinho, les risques de vol et d'agression ont considérablement aug- menté depuis qu'on a abandonné les contrôles systématiques des personnes qui y entrent. Les jeunes, marginaux ou non, se sont même depuis peu créé un véritable no man's land, appelé rocinha, sur le haut de la colline tout près, derrière l'hôtel Pelourinho, où il n'y a aucun contrôle policier et où les « cigares » de marijuana et autres substances illicites circulent en toute impunité.

En repoussant la marginalité sociale, juste aux limites du Pelourinho, on n'aura, en bout ligne, que contribué à la renforcer et à la rendre plus menaçante pour la population et les badauds de passage. En effet, avec la revitalisation, le Pelourinho est devenu un lieu qui génère beaucoup d'argent. Cette manne inespérée ne va pas sans susciter l'envie de plusieurs personnes marginales prêtes à tout pour avoir quelques reais pour payer, surtout, leur consommation de drogues. Le résultat le plus visible de cet effet pervers est certes le trafic de crack. Juste aux limites du Pelourinho, plus particulièrement dans la zone appelée Baixa do Sapateiro, se trouve la plus grande concentration de vente et d'usage de crack de toute la ville de Salvador. Tous les jeunes, que l'on voyait auparavant renifler des solvants, ont troqué leurs anciennes habitudes

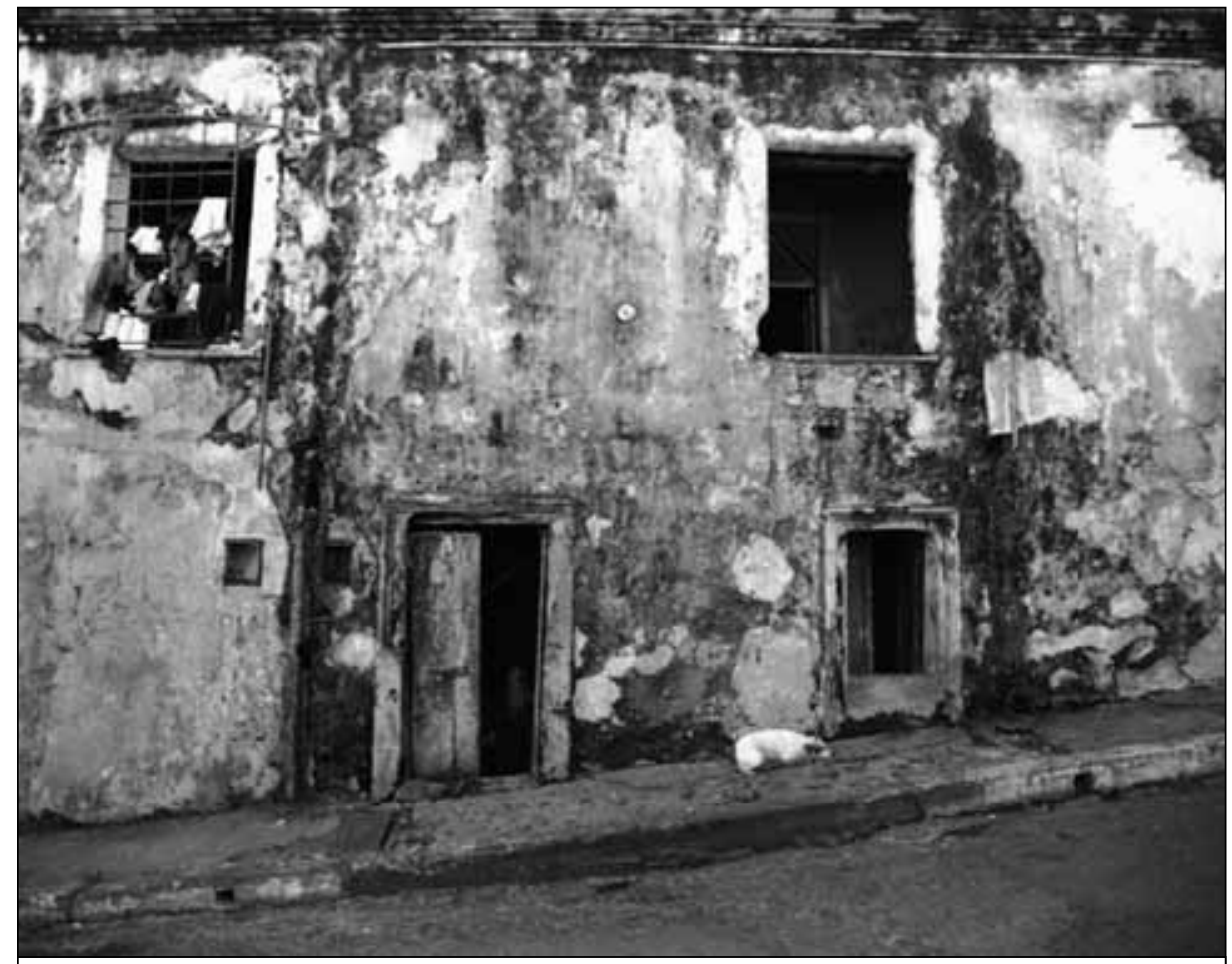

Périphérie du centre historique de Salvador de Bahia, Brésil.

Photo: Mélissa Robertson

pour la prise de pierre de crack. Les immeubles desquels ont été évincées les familles sont devenus des lieux de vente et de fumerie de crack. Pour se procurer cette drogue relativement bon marché, les jeunes de la rue n'ont qu'à mendier en simulant aux touristes passant la « faim terrible » qui les tenaille pour réussir, sans véritable difficulté, à leur soutirer les quelques reais nécessaires pour acheter leur dose de drogue. Les mères en manque, bébé à l'épaule, auront plus de facilité encore en demandant qu'on les aide à acheter du lait en poudre pour leur bébé, lait qu'elles revendront aussitôt pour se procurer leurs pierres de crack. En fait, la prise de crack dans le secteur atteint un niveau épidémique. Or, partout dans le monde où cette drogue de rue a fait des ravages, on assiste à une explosion de la violence urbaine. À Salvador, le problème de violence est d'autant plus aigu que, dans bien des cas, ce sont des policiers, qui gagnent des salaires dérisoires, qui assurent la "protection » des trafiquants sur la rue! Le monde de la prostitution est également de plus en plus violent, alors que prostitués et petits malfrats travaillent de mèche afin d'agresser les clients, tous, pour la plupart, des touristes amortis par la drogue que l'on aura glissée à leur insu dans leur verre... Ainsi, alors que l'on croyait pouvoir freiner l'expansion de la prostitution dans le secteur, n'a-t-on contribué indirectement qu'à rendre celle-ci plus violente. De plus, la présence dans les rues du Pelourinho d'une clientèle plus riche a entraîné l'arrivée de « prostituées de luxe », lesquelles étaient absentes avant la restauration.

Le portrait de la marginalité sociale dans les environs du Pelourinho est de plus en plus sombre. À cette violence en recrudescence, laquelle affecte sans distinction les plus pauvres comme les plus riches (la motivation égoïste de la consommation de crack étant plus forte que toutes les amitiés), s'ajoute la détérioration des conditions de vie et de santé des personnes marginales. La tuberculose est aujourd'hui endémique dans ces milieux, sans oublier le sida et les autres infections transmises sexuellement (ITS), telles que la syphilis, qui font un retour en force. Dans ce monde sans issu et sans avenir, le crack semble être le seul remède possible au mal de vivre de ces personnes. Mais pour combien de temps? 


\section{Quoi faire ? Une question toujours sans réponse}

Aujourd'hui, on ne peut penser qu'à réparer les pots cassés. II est toujours malaisé de commenter après coup les conséquences de décisions prises au détriment d'autres, dont on ne connaîtra jamais les résultats qu'elles auraient eus si elles avaient été appliquées. Nous pensons ici, surtout, au choix de faire du Pelourinho un endroit touristique sans habitants. On peut rétorquer que la détérioration du cadre bâti était telle que l'on n'avait pas d'autres choix que d'évincer les résidants pour leur propre protection. Mais, adopter une explication aussi simpliste, c'est faire le jeu des promoteurs du projet et des propriétaires sans vergogne qui ont laissé leur bâtiment à l'abandon en sachant qu'ils étaient de toute façon pour être grassement dédommagés sans devoir investir dans la restauration. En voulant faire du Pelourinho le cœur de la culture festive afro-brésilienne de Bahia, on s'est d'abord concentré sur l'aménagement physique des lieux. À ce chapitre, soulignons une autre fois que le résultat est magistral. L'idée d'inscrire l'imaginaire du petit monde de Jorge Amado dans l'espace physique, en baptisant entre autres plusieurs terrasses touristiques du nom de personnages ou de titres de ses romans, est également, croyonsnous, digne de mention. II reste que tout cela sonne faux dans cette vie dorénavant artificielle qui anime ce quartier peuplé de commerces à vocation touristique et d' "espaces culturels » voués à la culture festive afro-brésilienne ${ }^{16}$. Le Pelourinho est dorénavant un lieu physique où l'on se rend pour fêter et avoir du plaisir avec d'autres touristes, badauds ou visiteurs de passage comme soi. On ne s'y rend plus comme autrefois pour simplement partager la vie festive de ses habitants, ceux-ci faisant partie à leur tour du groupe de badauds.

D'aucuns diront qu'en évinçant les résidants du Pelourinho, on souhaitait nettoyer le secteur des marginaux sociaux qui avaient pris d'assaut les lieux pour en faire leur château-fort. On n'aura réussi en fait qu'à déplacer le problème un petit peu plus loin, mais pas assez loin pour empêcher les indésirables de venir y commettre leurs larcins ou y mener leurs activités délinquantes. En fait, on vient aujourd'hui d'encore plus loin dans la ville pour profiter de cette manne que représente la proie en apparence facile de touristes ou de riches fêtards. Les gens de la clase moyenne de Salvador le savent et ont de nouveau déserté les lieux qu'ils avaient pourtant pris plaisir à redécouvrir.
Les problèmes de marginalité sociale, y compris les problèmes de santé des personnes marginales elles-mêmes, sont aujourd'hui complexifiés par les nouveaux enjeux de violence qui affectent le centre historique. Le problème de l'usage et de l'accessibilité au crack, entre autres, est criant et ne peut se régler par la seule option de la répression.

Nous sommes à notre tour utopiste et rêvons d'un jour où marginaux, anciens habitants et promoteurs pourront s'asseoir ensemble pour examiner les solutions possibles aux problèmes sociaux et de santé qui découlent de l'actuelle occupation touristique du Pelourinho et de ses environs. Dans ce cas-ci, il est peut-être trop tard, car la frontière qui sépare les intérêts des plus pauvres et ceux des plus riches est de plus en plus éloignée, à l'image de la violence endémique qui oppose au Brésil les citoyens des favelas à ceux des quartiers riches. On ne peut toutefois que souhaiter que, avant de reproduire l'expérience ailleurs, l'on pense dès le départ à des moyens d'impliquer l'ensemble des " partenaires ", dont les habitants concernés, y compris les marginaux. II est toujours permis de rêver...

\section{Marc Perreault est docteur en anthropologie.}

\section{Notes}

1 Voir, entre autres, les statistiques de l'Organisation mondiale du tourisme (OMT) disponibles sur le site [www.world-tourism.org/ facts/menu.html]. Alors qu'en 1950 le nombre de voyageurs internationaux se chiffrait à 25 millions, en 2004 ils sont près de 763 millions, pour une croissance annuelle d'environ 6,5\% à l'échelle mondiale. Dans des régions telles que l'Asie et le Pacifique, cette croissance augmente jusqu'à $13 \%$ par année.

2 Autant par leurs conséquences sur les personnes que les milieux de vie, les phénomènes de marginalité sociale, de violence, en général, et de violence urbaine, plus spécifiquement, peuvent être traités sous une perspective de santé globale. Les gouvernements de pays démocratiques sont d'ailleurs de plus en plus enclins à adopter une approche de santé pour régler certains aspects de ces problèmes plutôt qu'à privilégier strictement l'approche répressive traditionnelle. Citons, plus près de nous, la politique de santé et de bien-être du Gouvernement du Québec de 1992 qui visait parmi ses principaux objectifs à diminuer de façon significative autant la délinquance, l'itinérance, que la violence familiale dans toutes ses extensions. Aussi croyons-nous qu'il est significatif, dans sa volonté d'ouverture démocratique, que le Brésil ait récemment inclus la « violence » parmi les catégories de problèmes de santé.
3 La restauration du cadre bâti est, en effet, souvent vue comme une solution aux problèmes de marginalité sociale, tels que la prostitution de rue, l'itinérance ou les « piqueries" qui affectent plus sévèrement certaines secteurs ou quartiers plus délabrés des grands centres urbains et non pas juste les zones touristiques. La logique derrière cette "solution » est que, dans les lieux plus désintégrés des villes, les formes de contrôles sociaux sont moins présentes, favorisant ainsi l'émergence et le maintien d'une population marginale qui trouverait dans ces lieux un milieu «naturel » de vie. Or, on croit qu'en restaurant le cadre bâti, en éliminant les zones plus désaffectées des quartiers, on rendra ces endroits moins favorables aux groupes de population plus pauvres et plus marginalisés socialement qui n'auront d'autres choix alors que de se diriger vers d'autres lieux. Au Québec, cette solution, privilégiée en particulier par les promoteurs immobiliers, participe du "syndrome" populaire appelé «Pas dans ma cour ». Sur ces problèmes de marginalité sociale et leurs solutions possibles, en lien principalement avec les enjeux des toxicomanies de rue, telles que vécues dans certaines zones urbaines du Québec, on peut se référer à quelques-uns de nos travaux de recherche (Bibeau et Perreault, 1995 ; Perreault, 1999 et 2000).

4 L'appellation populaire de la ville de Salvador a de quoi dérouter le touriste de passage. En effet, les habitants de Salvador, capitale de l'État de Bahia situé dans le nord-est du Brésil, ont I'habitude de dire qu'ils sont de Bahia ou Bahianais en se référant à la ville et non pas spécialement à l'État. Aussi, les personnes provenant de l'extérieur de la grande zone métropolitaine, aux limites du Reconcavo qui ceinture la baie de tous les saints, s'identifieront-elles plutôt comme étant de l'« intérieur », l'intérieur en question faisant référence dans l'imaginaire populaire à la fois à l'intérieur de l'État de Bahia et aux terres arides du sertão.

5 "A Bahia esta sempre em festa "; voir, par exemple: [http://www.bahia.com.br/]. Suivre le lien "Carnaval e festas " où il est écrit, entre autres : «La Bahia est toujours en fête. Ce sont des dizaines de fêtes qui se déroulent dans tout l'État durant l'année entière. Fêtes traditionnelles, folkloriques, religieuses, sportives, toutes marquées par l'allégresse caractéristique du peuple bahianais. "

6 Les principaux officiants du candomblé sont appelés, en fait, " père ou mère de saints " (pai de santos et mai de santos).

7 Sur l'importance des voyages entre Bahia et l'Afrique dans la consolidation actuelle du candomblé, on peut se référer entre autres à notre thèse de doctorat (Perreault, 2003) et au livre de Stefania Capone (1999). 
8 Pelourinho signifie littéralement « Pilori », de la place triangulaire du même nom -vestige de l'époque esclavagiste et de la lutte des Noirs pour leur libération - qui est située face à l'actuel musée Jorge Amado.

9 Pour l'anecdote touristique, soulignons que le lieu principal où se déroule l'action du roman Suor (68, de la Montée-du-Pelourinho) a aujourd'hui été transformé en hôtel intermédiaire et qu'une plaque commémorative rappelle au passant que c'est dans cet immeuble que vécurent les personnages de Suor.

10 "Ça semblait une vieille bâtisse comme les autres, écrasée dans la Montée-duPelourinho, coloniale, arborant des azuelos rares. Pourtant elle était immense. [...] Dans les cent seize chambres, plus de six cents personnes. Un monde. Un monde fétide, sans hygiène et sans morale, avec des rats, des jurons et des gens. Ouvriers, soldats, Arabes au langage estropié, colporteurs, voleurs, prostituées, couturières, portefaix, des gens de toutes les couleurs, de tous lieux, dans tous les costumes emplissaient la bâtisse. Ils buvaient de la cachaça au débit de Fernandes et crachaient dans l'escalier où, parfois, ils pissaient. Les uniques locataires gratuits étaient les rats. » (Amado, 1983: 14)

11 De cette visite est née la Fondation du patrimoine artistique et culturel de Bahia qui deviendra, plus tard, I'Institut du patrimoine artistique et culturel (IPAC), organisme paragouvernemental qui héritera de la gestion de l'ensemble du projet de préservation et de revitalisation du centre historique.

12 [http://whc.unesco.org/archive/advisory_body _evaluation/309.pdf].

13 Pour les détails de la situation de violence urbaine qui prévalait au Pelourinho au début de l'année 1991, on peut se référer à la description et à l'analyse que nous en faisons (Perreault, 2003: 21-28).

14 Sans entrer dans tous les détails de l'éviction des anciens habitants du Pelourinho et de ses environs, soulignons qu'on leur a offert au début deux possibilités en échange de leur déplacement, soit une relocalisation dans un autre quartier ou des compensations financières. Or, inutile de dire qu'en situation d'extrême pauvreté la grande majorité des ménages ont choisi la deuxième option puisque, de toute façon, la première était loin d'être assurée (Fernandes et al., 1995 : 34), si bien qu'une fois leur argent dépensé, la plupart des familles et des personnes n'avaient plus de quoi se reloger ailleurs. De plus, au fur et à mesure que le nombre des évincés grossissait, les possibilités de se relocaliser dans les environs diminuaient. Pour la première étape du projet de revitalisation, 399 familles se partagèrent environ 500000 \$US en indemnités, alors que seulement 26 familles optèrent pour leur déplacement dans des immeubles prédestinés; pour la deuxième étape, ce sont 176 familles qui se partagèrent environ 150000 \$US et 16 familles qui choisirent d'être relocalisées; pour la troisième étape, 374 familles se partagèrent environ 280000 \$US, tandis qu'aucune n'opta pour la relocalisation (IPAC, 1995 : 21). Précisons par ailleurs que la notion de famille est, ici, assez ambiguë (ou élastique) et qu'elle correspond au nombre de chambres ou de logements expropriés plutôt qu'au nombre réel de familles. Ainsi plusieurs personnes, voire même plus d'une famille, pouvaient habiter dans un même endroit, les personnes évincées ayant même avantage à grossir le nombre véritable des occupants afin d'augmenter la part de leur compensation.

15 Depuis la fin des années 1980, le reggae et l'allure rastafari prennent une place importante dans les représentations des jeunes et moins jeunes qui s'identifient à une certaine africanité. Or, la fête de la «Bénédiction des pains" qui a lieu tous les mardis soirs au Tereiro de Jesus et dans les rues du Pelourinho s'était transformée avec le temps en fête reggae. Jeunes et moins jeunes envahissaient les rues pour danser sur les airs de musique reggae. L'alcool coulait à flot et les gros cigares de marijuana passaient en toute liberté d'une main à l'autre durant cette fête improvisée de la rue. II est certain que de tels comportements n'étaient plus acceptables dans le nouveau Pelourinho. Aussi la fête du mardi, comme on l'appelle maintenant (A terça), a-t-elle été depuis complètement institutionnalisée et encadrée par les autorités municipales. N'ayant plus droit à la rue pour se manifester, les adeptes du reggae ont été déplacés à la fin des années 1990 dans une place entièrement fermée et clôturée en bas de la place du Pelourinho. Un tel déplacement n'a pas été sans engendrer de problèmes puisque, aujourd'hui, la place du reggae est certainement un des lieux les plus dangereux du Pelourinho: bandes de jeunes, petits malfrats et autres délinquants profitent de la promiscuité qui règne dans cet espace pour y venir tout autant régler leurs comptes que s'adonner à des larcins dont les touristes blancs, qui sont faciles à repérer, représentent la proie idéale. En restant cachée de la rue, cette violence est moins dérangeante pour l'image publique d'endroit sécuritaire que les autorités locales souhaitent diffuser à propos du Pelourinho, mais n'en demeure pas moins bel et bien réelle pour celui ou celle qui en écope.

16 À noter qu'après Olodum en 1991, nombreux sont les blocs carnavalesques qui sont venus installer leurs pénates dans les nouveaux locaux fraîchement rénovés du Pelourinho.

\section{Bibliographie}

Amado, Jorge (1983), Suor, Paris, Gallimard.

Bibeau, Gilles, et Marc Perreault (1995), Dérives montréalaises : À travers des itinéraires de toxicomanies dans le quartier HochelagaMaisonneuve, Montréal, Boréal.

Boutillier, Jean-Louis, Michèle Fiéloux, et Jean-Louis Ormières (1978), Le Tourisme en Afrique de l'Ouest. Panacée ou nouvelle traite? Paris, François Maspéro/Dossiers africains, p. 5-83.

Braga, Júlio Santana (coord.) (1969), Levantamento socioeconômico do Pelourinho. Salvador: Secretaria de Educação e Cultura do Estado da Bahia, Fundação do Patrimonio Artistico e Cultural da Bahia (recherche réalisée sous la direction de Vivaldo da Costa Lima).

Capone, Stefania (1999), La quête de l'Afrique dans le candomblé. Pouvoir et tradition au Brésil, Paris, Karthala.

Fernandes, Ana, et Marco Aurélio A. de Filgueira Gomes (1995) "Caminhos e descaminhos da requalificação do Pelourinho ", dans M. Meirelles et al. (dir.), Trilogia do Pelô, Salvador, Fundação Casa de Jorge Amado, p. 23-39.

Freyre, Gillberto (1974) [1933], Maitres et esclaves. La formation de la société brésilienne, Paris, Gallimard.

IPAC-Institut du patrimoine artistique et culturel (1995), Bahia. Centro histórico de Salvador, Programa de recuperação. Salvador, Corrupio.

Miranda, Luciete Barreto, et Maria Aparecida S.C. dos Santos (2000), Pelourinho: desenvolvimento socioeconômico, Salvador, Secretaria da Cultura e Turismo.

Perreault, Marc (1999), La ville et la toxicomanie: les enjeux communs de la désintégration urbaine, Rapport déposé au Comité permanent de lutte à la toxicomanie, 285 p. (synthèse du rapport non révisée par l'auteur accessible sur le site [www.cplt.com/publications/1099ville.pdf]).

Perreault, Marc (2000), Toxicomanie, mobilité dangereuse et vie de quartier, Actes du colloque « La vie itinérante ", Collectif de recherche sur l'itinérance, p. 51-58 [www.unites.uqam.ca/CRI/ communications/actes99/actePerrault.html].

Perreault, Marc (2003), Espaces, règles et jeux. Mythes intellectuels, "carnavalisation du monde " et invention de l'identité brésilienne, Thèse de doctorat en anthropologie, Université de Montréal, $380 \mathrm{p}$.

Verger, Pierre (1981), Noticias da Bahia - 1850, Salvador, Corrupio. 